\title{
Correction to: A handheld classroom dashboard: teachers' perspectives on the use of real-time collaborative learning analytics
}

\section{Roberto Martinez-Maldonado ${ }^{1}$}

Published online: 4 December 2019

(C) International Society of the Learning Sciences, Inc. 2019

\section{Correction to: International Journal of Computer-Supported Collaborative Learning} https://doi.org/10.1007/s11412-019-09308-z

The original version of this article unfortunately contained duplicate images for Figs. 4 and 6. The correct images are hereby published.

The original article has been corrected.

The online version of the original article can be found at https://doi.org/10.1007/s11412019-09308-Z

The online version of the original article can be found at https://doi.org/10.1007/s11412-019-09308-z

Roberto Martinez-Maldonado

Roberto.MartinezMaldonado@Monash.edu

1 Faculty of Information Technologies, Monash University, Melbourne, VIC, Australia 


\section{Visualisation about task progress Visualisation about individual participation}

\section{3 ideas}

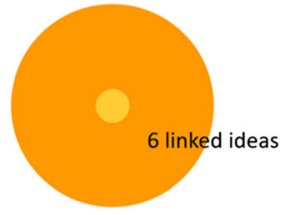

Cong



Yuchao

Fig. 4 Visualisations about group task progress (left- represented as two concentric circles whose area represents the size of the group solution and the distance to the teacher's solution for the outer and inner circles respectively) and individual participation (right-spider diagram of the amount of touch interaction per student)

Class script timeline visualisation

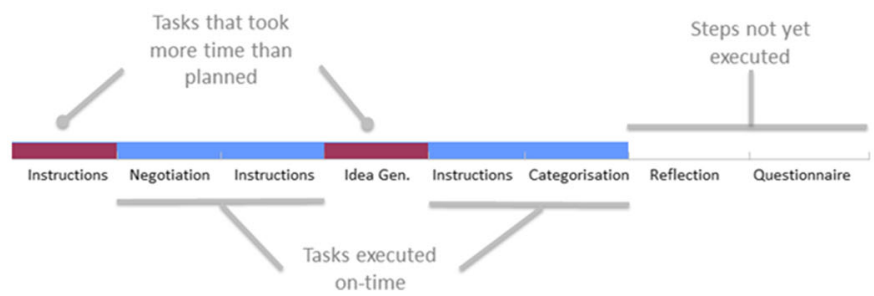

Timer alarm

when the current task is on-time

4:23
out of $7: 00$

when the current task is taking more time than planned

$7: 25$

out of $7: 00$

Fig. 6 Real-time feedback on the teacher's enactment of the learning design in the form of a timeline visualisation (left) and an explicit timer alarm (right) 